\title{
Erythema multiforme caused by tattoo: a further case
}

\author{
Attilio Allione $\cdot$ Luca Dutto $\cdot$ Elisabetta Castagna $\cdot$ \\ Marco Ricca $\cdot$ Bruno Tartaglino
}

Received: 22 October 2009/Accepted: 27 March 2010/Published online: 22 June 2010

(C) SIMI 2010

\section{Introduction}

Since the increasing popularity of tattoos among young people, numerous and variegated complications are described. For several years, para-phenylenediamine (PPD) has been used to darken the henna dye commonly used for tattoos. PPD is a potent additive sensitizer and contact dermatitis is the most common reaction pattern.

Erythema multiforme (EM)-like reaction secondary to PPD henna tattoo contact is a well described but unusual manifestation, and only six previous cases are reported [1-3].

\section{Case report}

A 20-year-old woman had a henna tattoo applied to her right upper arm. Two days later an eczematous rash developed at the tattoo site (Fig. 1) Further, 3 days later she was admitted to the hospital because of a multiforme erythema localized to the legs bilaterally. She was otherwise well, with no dyspnea, cough or pleuritic pain. She had no history of recent illness, in particular viral illness, or drug intake. No topical ointment or systemic antibiotic were administrated after placement of the tattoo.

On examination, the vital signs were: temperature $37.0^{\circ} \mathrm{C}$, blood pressure $110 / 78 \mathrm{mmHg}$, heart rate 100 beats per minute. She had no skin or scleral jaundice. There was no lymphadenopathy. The physical examination was otherwise normal. The erythema with the characteristic

A. Allione $(\bowtie) \cdot$ L. Dutto $\cdot$ E. Castagna $\cdot$ M. Ricca .

B. Tartaglino

Department of Emergency, Santa Croce and Carle Hospital,

Via Michele Coppino 26, 12100 Cuneo, Italy

e-mail: allione.attilio@libero.it annular target-like shape at the site of the tattoo was severe, sparing the area immediately adjacent to the tattoo. Similar lesions appeared on bilateral pre-tibial areas (Fig. 2).

The white-cell count was $9,800 / \mathrm{mm}^{3}$. The hemoglobin level was $12.8 \mathrm{~g} / \mathrm{dl}$, with a normal mean corpuscular volume. The hematocrit was $36.5 \%$, and the platelet count was $270,000 / \mathrm{mm}^{3}$. She had no eosinophilia. Renal and liver function tests were normal. The serum electrolyte levels were normal. The C-reactive protein level was mildly elevated at $25 \mathrm{mg} / \mathrm{l}$ (normal value $<10 \mathrm{mg} / \mathrm{l}$ ). A chest $\mathrm{X}$-ray film revealed no abnormalities or pleural effusion. Patch tests were not performed because of the concerns about the risk of precipitating a severe reaction to PPD.

Systemic steroids were prescribed and the lesions healed completely in about 1 week.

\section{Discussion}

Our skin as a canvas for self-expression, identity and individualism through body art (including tattoos, piercings, and scarification) represents an old practice that has become very popular in the present era.

Numerous complications due to dermal tattooing, including pyogenic infections, viral hepatitis, syphilis, tuberculosis cutis, rubella, herpes simplex, herpes zoster, psoriasis, lichen planus, lupus, pigment allergy and sensitivity, keloids, sarcoidal granulomas, endophthalmitis, malignant melanoma, squamous cell carcinoma, and basal cell carcinoma, have been described.

Tuberculosis cutis is a challenge in the Emergency Department (ED). However, our patient was at low risk for the development of tuberculosis (TBC) because she lives in the North West of Italy, in a non-TBC endemic area. Moreover, there were no elements in this case that 


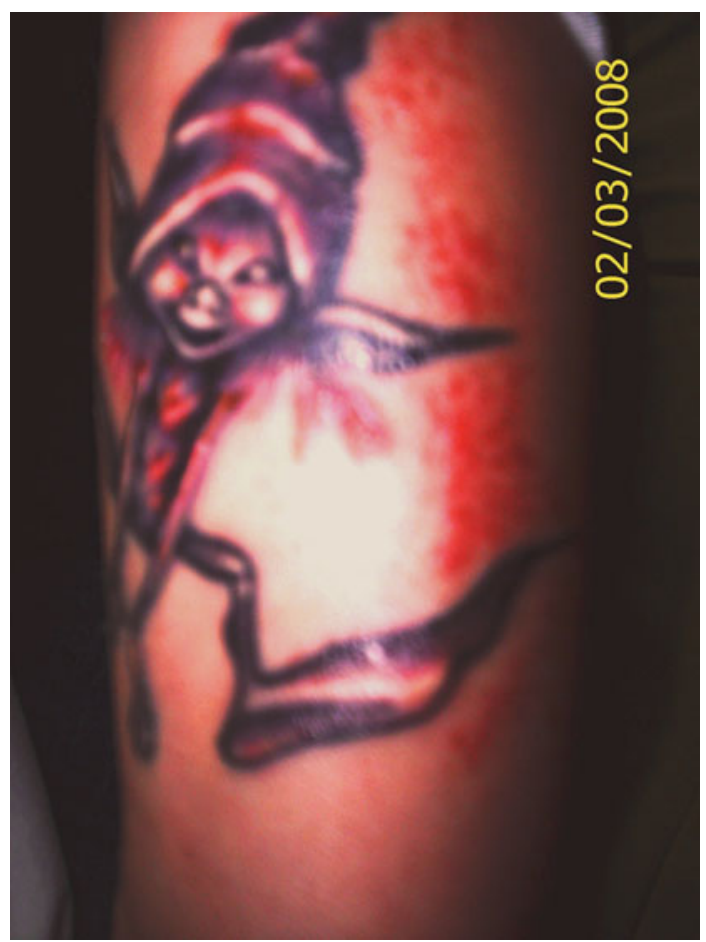

Fig. 1 Localized eczematous rash within the site of tattoo

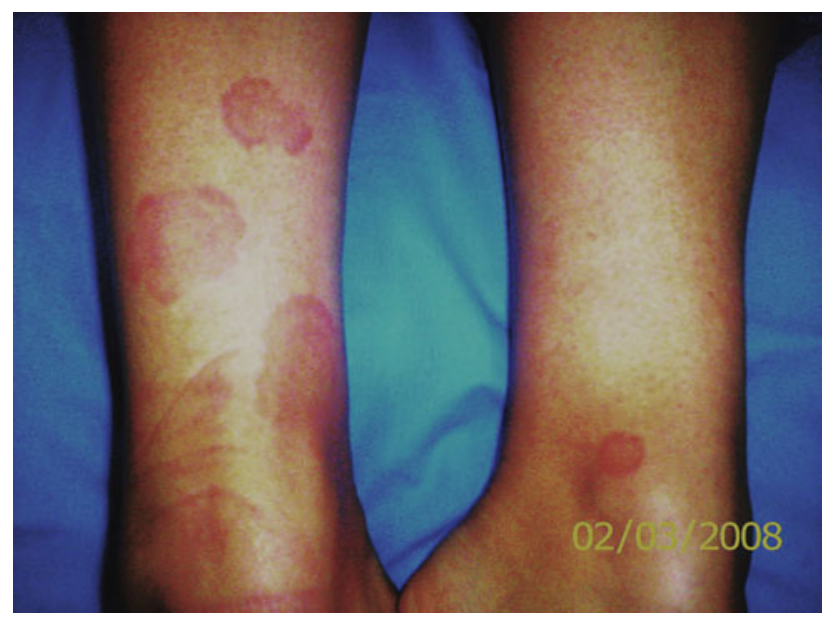

Fig. 2 The erythema with the characteristic annular target-like shape on bilateral pre-tibial areas

suggested a possible contact with a contagious adult, and the patient was deemed not to be at risk for tuberculosis.

Henna is obtained from the leaves of the Lawsonia inermis tree, native of Sri Lanka, India and North Africa. Henna is used to darken temporary tattoos, together with a mixture of other substances like $p$-phenylenediamine (PPD), a derivative of analine, that is a powerful sensitizer. Despite the geographic provenance, temporary henna tattoos have become increasingly widespread in Europe, especially among children and teenagers. The practice is popular during holiday periods, thanks to its rapid execution, inexpensive costs, and the presence of seasonal specialized artists.

Reaction patterns to PPD include angioedema and an eczematous reaction, but an unusual manifestation of allergic contact sensitivity can be an EM-like reaction. Our patient had no known history of exposure to PPD.

Previously, rare occurrences of EM-like reactions to PPD and its derivatives have been described [1-3].

The classic feature of EM-like reactions is the presence of red, annular lesions, with a target-like, or bull's-eye. The rash usually appears first near the contact dermatitis with sparing of the immediately adjacent skin, and then it generalizes [4].

Lyme disease should be considered in a young person with these changes in the skin. This diagnosis is improbable, however, since examination did not reveal a tick bite, and since the systemic symptoms include low-grade fever, malaise, arthralgias, headache and lymphadenopathy, which were not present in our case. Bullous EM-like contact dermatitis is rarely reported.

The mechanism of EM secondary to contact dermatitis is unknown, but is thought to involve a type III reaction due to immune complex formation. Another possible mechanism involves a delayed hypersensitivity (type IV) reaction seen in eczematous allergic contact dermatitis.

Even today, infectious complications are still possible because of insufficient personal hygiene or lack of hygiene control in the tattoo parlor. Moreover, especially in the initial phases, the differentiation between EM-like and local infection diagnoses may well be impossible. In general, local infections are only located in the tattooed area and an increased risk of infections is present when concomitant diseases exist [5].

Topical antibiotic ointment or certain local anesthetics are sometimes used by tattoo artists. A hypersensitivity reaction may occur, but in this case no topical ointment or antibiotic were administrated after placement of the tattoo. A complete blood count and differential count, with specific consideration of eosinophilia as a potential marker of hypersensitivity, would be an important starting point for the evaluation [5].

Because of the increase in the number of people obtaining tattoos, among the numerous complications of dermal tattooing, the EM-like reactions should be included in the differential diagnosis.

\section{Conflict of interest None.}

\section{References}

1. Jappe U, Haunsen BM, Petzholdt D (2001) Erythema-multiformelike reaction and depigmentation following allergic contact dermatitis froma a paint-on henna tattoo due to paraphenylenediamine contact hypersensitivity. Contact Dermat 24:249-250 
2. Neri I, Guareschi E, Savoia S et al (2002) Childhood allergic contact dermatitis from a henna tattoo. Pediatr Dermatol 19:503-505

3. Sidwell RU, Francis ND, Basarab T et al (2008) Vesicular erythema multiforme-like reaction to para-phenylenediamine in a henna tattoo. Pediatr Dermatol 25:201-204
4. Torinuki W (1990) Generalized erythema-multiforme-like eruption following allergic contact dermatitis. Contact Dermat 23:202-203

5. Kazandjiev J, Tsankov N (2007) Tattoos: dermatological complications. Clin Dermatol 25:375-382 